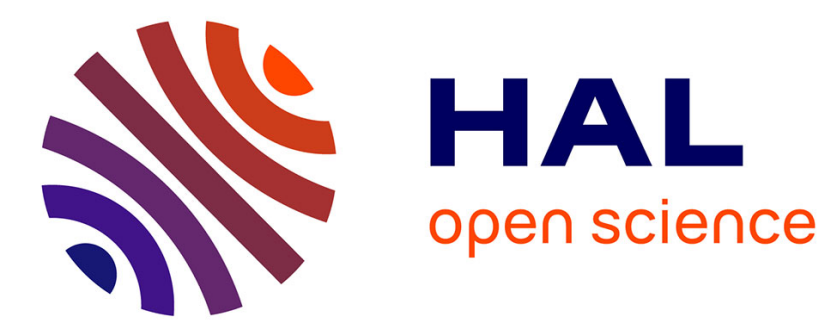

\title{
Les bobinages et le stockage d'énergie
}

M. Sauzade, J. Solé

\section{To cite this version:}

M. Sauzade, J. Solé. Les bobinages et le stockage d'énergie. Revue de Physique Appliquée, 1969, 4 (4), pp.581-582. 10.1051/rphysap:0196900404058100 - jpa-00243337

\section{HAL Id: jpa-00243337 https://hal.science/jpa-00243337}

Submitted on 1 Jan 1969

HAL is a multi-disciplinary open access archive for the deposit and dissemination of scientific research documents, whether they are published or not. The documents may come from teaching and research institutions in France or abroad, or from public or private research centers.
L'archive ouverte pluridisciplinaire HAL, est destinée au dépôt et à la diffusion de documents scientifiques de niveau recherche, publiés ou non, émanant des établissements d'enseignement et de recherche français ou étrangers, des laboratoires publics ou privés. 


\title{
LES BOBINAGES ET LE STOCKAGE D'ÉNERGIE
}

\author{
M. SAUZADE et J. SOLÉ.
}

$\mathrm{Au}$ cours de cette table ronde ont été évoqués les différents problèmes posés par le stockage et la libération de l'énergie magnétique emmagasinée dans une bobine supraconductrice. Ces problèmes sont différents suivant que l'énergie est libérée rapidement ou lentement. Ces deux aspects ont été abordés et différentes solutions ont été proposées. Un problème particulier : celui de la stabilisation des matériaux supraconducteurs, qui dépasse largement le cadre de l'application au stockage, a été longuement débattu. Une discussion animée a montré qu'il était loin d'être parfaitement résolu. Pour clore cette table ronde, quelques caractéristiques de la plus grosse bobine construite actuellement en France ont été données.

Stockage d'énergie. - Le stockage d'énergie sous forme magnétique présente l'avantage de permettre une très grande densité d'énergie dans un « diélectrique » peu coûteux : l'air. Cette densité atteint $40 \mathrm{~kJ}$ au litre en utilisant les matériaux supraconducteurs actuels. Elle est environ 100 fois plus grande que dans un condensateur. Un bilan économique montre qu'audelà de $100 \mathrm{~kJ}$ l'utilisation d'un bobinage supraconducteur est plus avantageux que l'emploi de condensateur. Une particularité du stockage magnétique peut s'avérer intéressante dans certaines applications, c'est la loi de décharge qui impose un courant maximum. Dans le cas d'un plasma par exemple, il y a autorégulation de la décharge, car lorsque la résistance de l'arc croît, l'augmentation de la tension de la source qui en résulte tend à s'opposer à la variation de courant.

L'énergie emmagasinée peut être utilisée dans une décharge rapide (quelques microsecondes à quelques secondes) ou dans une décharge lente pouvant atteindre plusieurs heures. Les problèmes à résoudre sont alors très différents.

- Dans les décharges rapides, le bobinage supraconducteur est utilisé comme un transformateur de puissance. La puissance instantanée obtenue au moment de la décharge dépasse souvent la centaine de mégawatts. Le problème important à résoudre est évidemment celui de l'interrupteur qui permettra une telle décharge. Diverses solutions ont été proposées.

Un premier moyen (C.E.A. Limeil) consiste à utiliser un interrupteur que l'on fait passer de l'état supraconducteur à l'état normal. Le passage entre les deux états, qui peut être de l'ordre de $1 \mu$ s, est obtenu en portant le supraconducteur au-dessus de sa température critique ou au-dessus de son champ critique, éventuellement même en superposant les deux actions. L'utilisation d'un interrupteur de ce type présente toutefois une difficulté, car la faible résistivité à l'état normal des matériaux supraconducteurs usuels oblige à utiliser de grandes longueurs de supraconducteur pour que l'interrupteur présente une résistance élevée devant l'impédance de charge. Le volume de l'interrupteur devient rapidement important pour de très grandes puissances.

Pour ce type d'application, il y aurait lieu de développer des matériaux supraconducteurs à forte densité de courant critique dont la résistivité à l'état normal soit très élevée.

Une deuxième solution plus classique (Institut d'Électronique d'Orsay) consiste à utiliser un interrupteur mécanique sous vide placé à l'extérieur de l'enceinte cryogénique. Ce type d'interrupteur n'est pas capable de couper un courant continu de forte intensité, car l'écartement des électrodes est si faible (environ $8 \mathrm{~mm}$ ) qu'une tension de $20 \mathrm{~V}$ suffit à entretenir un arc. Il présente toutefois l'avantage d'avoir un temps de désionisation extrêmement court (environ $1 \mu \mathrm{s}$ ) après annulation du courant. L'interrupteur s'ouvre donc très rapidement si l'on fait passer artificiellement le courant par zéro. Pour obtenir ce résultat, on décharge dans l'interrupteur un condensateur convenablement polarisé à l'aide d'un circuit annexe.

Ce dispositif a permis de couper des intensités de 450 A en une milliseconde avec des surtensions à l'ouverture de l'ordre de $23 \mathrm{kV}$.

- Dans le cas d'une décharge lente, la bobine est utilisée comme source d'énergie. Une étude prospective (E.D.F., Fontenay-aux-Roses) montre par exemple qu'il pourrait être rentable de réaliser un bobinage de stockage permettant de faire l'économie d'une centrale de pointe. Pour couvrir les pointes de consommation d'une grande ville, il faut une énergie d'environ $10^{13} \mathrm{~J}$. Le bobinage nécessaire pour stocker une telle énergie aurait un diamètre extérieur de $102 \mathrm{~m}$ et un diamètre intérieur de $20 \mathrm{~m}$.

Stabilisation des matériaux supraconducteurs. Pour utiliser les matériaux supraconducteurs dans les bobinages, il est nécessaire de les stabiliser en les 
recouvrant d'un revêtement en métal normal de faible résistivité. Lors d'une transition d'une partie du supraconducteur à l'état normal (sous l'influence d'un saut de flux par exemple), le courant circule en majeure partie dans le revêtement où il provoque un échauffement local. Si le conducteur est bien refroidi, l'énergie dissipée par effet Joule est évacuée rapidement par le bain d'hélium liquide; l'échauffement n'est pas suffisant pour altérer les propriétés du supraconducteur (courant critique et champ critique).

Dès la disparition du phénomène qui a provoqué la transition, le matériau retourne à l'état supraconducteur. On dit que la stabilisation est complète.

Si le matériau n'est pas suffisamment stabilisé, l'élévation locale de la température est suffisante pour dégrader ses propriétés. Dans un champ magnétique donné, il faudra diminuer le courant que supporte le conducteur pour qu'il retrouve ses propriétés supraconductrices. Le courant pour lequel le matériau redevient supraconducteur est appelé « courant de récupération ». Il caractérise le degré de stabilisation $\mathrm{du}$ conducteur.

La qualité de la stabilisation dépend de la résistivité du métal utilisé pour le revêtement, de sa conductibilité thermique, de sa chaleur spécifique et de la résistance thermique et électrique de contact entre le matériau supraconducteur et le revêtement. Elle est aussi fonction de la qualité des échanges thermiques avec le bain d'hélium.

Différentes études expérimentales sont faites en France en utilisant comme revêtement du cuivre (Thomson-C.S.F.) ou de l'aluminium (C.G.E.). L'aluminium commercial présente une résistivité plus faible que le cuivre à basse température, par contre sa tenue mécanique est nettement inférieure. Les résis- tances thermique et électrique de contact semblent aussi moins bonnes dans le cas de l'aluminium.

A l'heure actuelle, il est difficile de mettre en évidence des avantages certains d'un matériau par rapport à l'autre.

Une étude théorique de la stabilisation est actuellement réalisée à l'Alsthom pour examiner l'importance des différents paramètres qui rentrent en jeu.

Il est certain que ces études sont d'une grande importance pour les applications. On connaît mal actuellement l'influence des différents paramètres et la mesure du courant de récupération ne donne qu'un critère pratique global. Il serait d'un grand intérêt de réaliser des revêtements à forte chaleur spécifique qui limiteraient l'élévation de température. On pourrait pour cela utiliser des matériaux ayant une transition de phase à une température légèrement supérieure à celle de l'hélium liquide.

On peut par ailleurs chercher à réaliser un matériau intrinsèquement stable dont le courant critique croisse lorsque la température s'élève. Ce résultat pourrait être atteint en provoquant, par l'élévation de température, une augmentation des centres de piégeage.

Bobinage pour chambre à bulles. - Le Centre Nucléaire de Saclay a réalisé un bobinage de grandes dimensions pour chambre à bulles. Il est constitué de deux bobines de $1 \mathrm{~m}$ de diamètre en position d'Helmholtz. Le champ magnétique au centre est de $40 \mathrm{kG}$. L'énergie stockée de $10 \mathrm{MJ}$. L'une des bobines est réalisée à l'aide d'un ruban supraconducteur de fabrication française (Thomson-C.S.F.), l'autre de fabrication anglaise (I.M.I.). Le ruban de section rectangulaire supporte une intensité de $1750 \mathrm{~A}$ à $54 \mathrm{kG}$. La densité de courant est de $50 \mathrm{~A} / \mathrm{mm}^{2}$. 